\title{
Responsabilidad social de las PyMEs en el marco del posconflicto, como soporte para la inclusión laboral de la población reinsertada
}

FECHA DE RECEPCIÓN: 20 de agosto de 2015

FECHA DE APROBACIÓN: 10 de noviembre de 2015

Pp. 45-61
María Elsy Sepúlveda Romero* Edna Piedad Moreno Martínez**

Julián Eduardo Tovar Mesa**

Jairo Andrés Franco Villada**

Cindy Lizeth Villarraga Tole**

\section{Resumen}

Un gran porcentaje del tejido empresarial en Colombia son las PyMEs, donde la responsabilidad social tarda en llegar, muchas veces por desconocimiento, otras por no realizar determinadas tareas de otra forma y en otras ocasiones lo que hacen no se comunica. Objetivos: Establecer la responsabilidad social de las PyMEs en el marco del posconflicto; determinar si las éstas conocen las políticas gubernamentales que incentivan a las empresas en la generación de empleo para la población reinsertada y precisar si cuentan con políticas de RSE. Métodos: La investigación es de tipo cuantitativo descriptivo, la población objeto de estudio son 20 microempresas ubicadas en la ciudad de Bogotá, se seleccionó como instrumento de recolección de datos; una encuesta, en la que se plantearon seis preguntas cerradas. Resultados: las PyMEs no conocen las políticas gubernamentales que promueven, apoyan o incentivan la generación de empleo para las personas reinsertadas; las PyMEs están comprometidas casi por completo con la oferta de empleo para los desmovilizados de las FARC y su aporte en el posconflicto, incentivan su decisión de contratar personal reinsertado, si el gobierno implementa estímulos tributarios y aquéllas que fueron encuestadas están dispuestas a trabajar en conjunto con el gobierno para diseñar políticas de RSE que busquen mejorar la calidad de vida y propiciar ambientes sociales adecuados para la población reinsertada en el marco del posconflicto.

Discusión: El gobierno no ha hecho un esfuerzo suficiente en involucrar a las PyMEs en el compromiso de generación de políticas de RSE y en la preparación para la oferta de empleo en el posconflicto. Conclusión: Se establece que la mayoría de las PyMEs no tienen políticas de RSE y algunas de ellas no saben qué es, pero muchas la ejecutan de forma indirecta, capacitando a los empleados, cumpliendo todos los compromisos con los grupos de interés y sobretodo, aportando al medio ambiente y a los problemas sociales, como es la inclusión laboral de la población reinsertada en el marco del posconflicto.

\section{Palabras clave}

Responsabilidad social, PyMEs, posconflicto, inclusión laboral y población reinsertada.

* Magíster en Educación, Universidad Externado de Colombia. Especialista en Supervisión y Control, Universidad Externado de Colombia.

** Estudiantes décimo semestre de Administración de Empresas, Universidad EAN. 


\title{
Social responsibility of small and medium enterprises within the post-conflict context, as a support for labor inclusion of reinserted population
}

\begin{abstract}
A large percentage of the business sector in Colombia are the Small and Medium Enterprises (SMEs), where social responsibility is usually delayed, often due to unawareness, or for not performing certain tasks differently, and sometimes what they do is just not communicated. Goals: to establish the social responsibility of SMEs in the context of post-conflict; to determine if SMEs know the government policies that encourage companies to generate employment for the population who have been reintegrated into society, and to indicate whether SMEs have Corporate Social Responsibility (CSR) policies. Methods: this research is of the quantitative descriptive type, the study population is 20 micro-enterprises located in Bogotá, and a survey with six closed questions was selected as the instrument for data collection. Results: the SMEs are not aware of government policies that promote, support or encourage the creation of jobs for people who have been reintegrated into society; SMEs are almost entirely engaged with job openings for persons who are demobilized from the Revolutionary Armed Forces of Colombia (FARC for its abbreviation in Spanish) and their contribution in post-conflict. SMEs encourage the government's decision of hiring people who have been reintegrated into society only if it implements tax incentives. Surveyed SMEs are willing to work together with the government to design CSR policies that seek to improve quality of life and foster adequate social environments for reinserted people in the context of post-conflict.

Discussion: the government has not made enough effort to involve SMEs in the commitment of generating CSR policies and in the preparation of job offers in the post-conflict. Conclusion. It is established that most SMEs have CSR policies and some of them do not know what CSR is, but many of them execute it indirectly, training employees, meeting all commitments with stakeholders and especially contributing to environment and social issues, such as labor inclusion of people who have been reinserted into society in the context of post-conflict.
\end{abstract}

\section{Keywords}

Social responsibility, Small and Medium Enterprise (SME), post-conflict, labor inclusion, reinserted population. 


\section{Responsabilité sociale des pme colombiennes dans le cadre de l'après-conflit: une plateforme pour la réinsertion professionnelle de la population}

\section{Résumé}

Les PME colombiennes représentent un large pourcentage du tissu entrepreneurial national mais leur sens de la responsabilité sociale tarde à se mettre en place, souvent par ignorance des procédures, mais aussi car ce qu'elles mettent en place n'est pas toujours porté à la connaissance du grand public. Objectifs: Établir la responsabilité sociale des PME dans le cadre de l'après-conflit; déterminer si les PME connaissent les politiques gouvernementales facilitant aux entreprises la création d'emploi spécifiques à la population réintégrée à la société civile et enfin savoir si les PME disposent de politiques efficaces de responsabilité sociale et entrepreneuriale. Méthode: L'investigation est de type quantitatif et descriptif ; l'objet de l'étude se compose de 20 micro-entreprises situées dans la ville de Bogotá; la méthode sélectionnée pour la collecte des données a été l'enquête individuelle comprenant six questions fermées. Résultats: Les PME ne connaissent pas les politiques gouvernementales pour la promotion et l'aide au retour à l'emploi des personnes réintégrées à la société civile; les PME soutiennent dans leur immense majorité le retour à l'emploi de la population démobilisée de la guérilla des FARC et leur apport dans la société; les PME réitèrent leur disposition à embaucher des anciens combattants des FARC si le gouvernement met en place des mesures d'aide fiscale; elles sont également disposées dans l'ensemble à travailler avec le gouvernement colombien pour créer des politiques de responsabilité sociale et entrepreneuriale contribuant à améliorer la qualité de vie des anciens combattants en favorisant la création d'un climat social approprié.

Discussion: Le gouvernement n'a pas réalisé suffisamment d'efforts pour davantage impliquer les PME dans la création de politiques de responsabilité sociale. Conclusion: Il est établi que la majorité des PME ne disposent pas de politiques de responsabilité sociale ou entrepreneuriale et certaines en ignorent même l'objet. Cependant un nombre important de PME réalisent une forme de responsabilité sociale de manière indirecte, en formant leurs employés, en s'acquittant de tous les engagements des groupes d'intérêt et surtout en contribuant à la protection de l'environnement et à la résolution des conflits sociaux, comme peut l'être la réinsertion professionnelle de la population démobilisée dans le cadre de l'après-guerre.

\section{Mots clefs}

Responsabilité sociale, PME, Gestion de l'après-guerre, Réinsertion professionnelle, Population démobilisée. 


\section{Responsabilidade social das PyMEs no marco do pós-conflito, como suporte para a inclusão laboral da população reinserida}

\section{Resumo}

Uma grande porcentagem do tecido empresarial na Colômbia são as PyMEs, onde a responsabilidade social tarda em chegar, muitas vezes pelo desconhecimento, outras por não realizar determinadas tarefas de outra forma e em outras ocasiões o que fazem não se comunica. Objetivos. Estabelecer a responsabilidade social das PyMEs no marco do pós-conflito; determinar se as PyMEs conhecem as politicas governamentais que incentivam as empresas na geração de emprego para a população reinserida e precisar se as PyMEs contam com politicas de SER. Métodos. A pesquisa cientifica é de tipo quantitativo descritivo, a população objeto de estudo são 20 microeempresas localizadas na cidade de Bogotá, se selecionou como instrumento de coleta de dados uma pesquisa, na qual se apresentaram seis perguntas fechadas. Resultados. As PyMEs não conhecem as políticas governamentais que promovam, apoiem ou as incentive na geração de emprego para as pessoas reinsertadas; as PyMEs estão comprometidas quase por completo com a oferta de emprego para os desmobilizados das FARC e seu aporte no pós-conflito, as PyMEs incentivam sua decisão de contratar pessoal reinserido, se o governo implementa estímulos tributários e as PyMEs pesquisadas estão dispostas a trabalhar em conjunto com o governo para projetar politicas de SER que busquem melhorar a qualidade de vida e proporcionar ambientes sociais adequados para a população reinserida no marco do pós-conflito.

Discussão. O governo não fez um esforço suficiente em envolver as PyMEs no compromisso de geração de políticas de SER e na preparação para a oferta de emprego no pós-conflito. Conclusão. Estabelece-se que a maioria das PyMEs não têm políticas de SER e algumas delas não sabem o que é SER, porém muitas a executam de forma indireta, capacitando aos empregados, cumprindo com todos os compromisso com os grupos de interesse e sobretudo aportando ao meio ambiente e aos problemas sociais, como é a inclusão laboral da população reinserida no marco do pós-conflito.

\section{Palavras-chave}

Responsabilidade social, PyMEs, pós-conflito, inclusão laboral e população reinserida. 


\section{Introducción}

ctualmente Colombia vive un proceso de desmovilización, dentro del cual
hay un programa de reintegración social, en donde los desmovilizados logran vincularse y reingresar a la sociedad civil a través de diferentes canales. Uno de estos canales es la inclusión laboral de estas personas mediante la figura de la Responsabilidad Social Empresarial, sin embargo, esta política ha sido muy compleja, pues existe una tendencia a imponer una barrera por parte de las empresas del sector privado de vincular personas desmovilizadas dentro de su nómina. (Roldán, 2013).

El concepto de Responsabilidad Social Empresarial (RSE) ha cambiado desde lo expuesto por Friedman, quien relacionaba la responsabilidad de la empresa únicamente con la generación de utilidades y el cumplimiento de la normatividad establecida, luego pasó a una visión en donde se vinculan las partes interesadas y se habla de una ética empresarial (Backman, 1970) y por último - se incluye en el concepto RSE - el que la empresa se involucre en los problemas sociales y el cuidado al medio ambiente.

Sin embargo, y en el caso de las PyMEs, existe una falsa creencia de que la Responsabilidad Social Empresarial es exclusiva de las grandes empresas, siendo esto una de las principales barreras que al momento de acercarse a la responsabilidad empresarial como una nueva forma de hacer negocios (González, 2011).

Dadas las potenciales condiciones de las PyMEs, se realizó el presente estudio, con la finalidad de establecer la responsabilidad social de las PyMEs en el marco del posconflicto; determinar si las PyMEs conocen las políticas gubernamentales que incentivan a las empresas en la generación de empleo para la población reinsertada y tercero, precisar si las PyMEs cuentan con políticas de RSE.

El presente artículo se inicia con la definición del concepto de RSE y de inclusión laboral de población reinsertada a partir de diferentes planteamientos; luego, se abordarán los métodos utilizados en el estudio; en tercer lugar se plantean los resultados producto de la encuesta realizada; en cuarto lugar, se efectúa la discusión generada por los resultados y por último, se concluye. 


\section{Responsabilidad social empresarial}

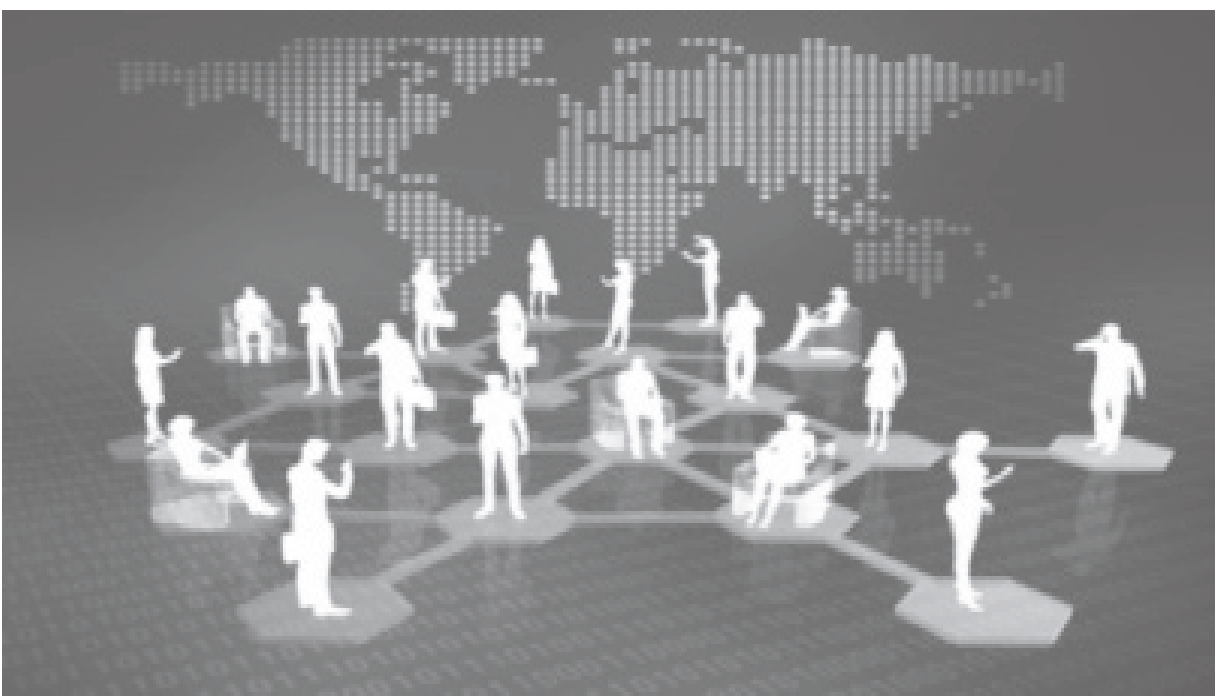

Fuente. Inercia digital. (2015).

La Responsabilidad Social Empresarial, en adelante RSE, tiene su fundamento primario en el Derecho Internacional, específicamente en el Libro Verde de Responsabilidad Social de la Comisión Europea, publicado en el año 2001.

Para la Comisión Europea (2001), la Responsabilidad Social en una empresa consiste de forma voluntaria, integrar dentro de las operaciones comerciales y las relaciones con sus grupos de interés, los problemas sociales y medioambientales, afirmando, que una empresa no es socialmente responsable únicamente cuando cumple con sus obligaciones jurídicas, sino que debe ir más allá, pues debe aportar más en capital humano, en el entorno y en las relaciones con los actores sociales que intervienen en estos procesos.

Por otro lado, Cajiga (2011) sostiene que la Responsabilidad Social Empresarial es un compromiso que tiene toda empresa de cumplir con su finalidad, en la cual, se incluyen los aspectos económicos, sociales y ambientales y en donde participan los grupos de interés, con el fin de contribuir a la construcción del bien común. 
Ahora bien, con relación a la observancia de la Responsabilidad Social Empresarial en Colombia, esta se enfoca a partir de diversos aspectos como son: la preocupación por la violación de los derechos humanos, los impactos negativos en el medio ambiente, el desmejoramiento de la calidad de vida de la población, los escándalos de corrupción y el trabajo infantil, entre otros, por lo cual, Colombia se adhirió a la Declaración del Milenio del año 2000, que incluye ocho objetivos para ser cumplidos a partir del presente año, entre ellos se encuentran: combatir el hambre y la pobreza extrema; dar educación; igualdad entre géneros; bajar la mortalidad de los niños; sostenibilidad del medio ambiente.

Al respecto conviene decir que en Colombia existe el Centro Colombiano de Responsabilidad Empresarial (CCRE), que es la organización encargada del estudio de la RSE y quién define la RSE como aquella capacidad de respuesta que tiene toda empresa frente a las implicaciones de sus acciones sobre los diferentes grupos de interés con los que se relaciona, de ahí que, las empresas son socialmente responsables cuando las actividades que realiza se enfocan a satisfacer las necesidades y expectativas de la sociedad, así como también, al cuidado y preservación del entorno (CCRE, 2004).

Por otro lado, en el año 2008 el ICONTEC ratificó el documento Guía Técnica de Responsabilidad Social - GTC 180, cuya aplicación es de carácter voluntario, el cual, facilita las pautas para establecer, implementar, mantener y mejorar de forma continua un enfoque de responsabilidad social en la gestión, tratando de involucrar a los grupos de interés en dicha orientación.

Cabe señalar que en el año 2005 se desarrolló la ISO 26000, documento expedido por el Organismo Internacional de Normalización que ofrece a las empresas orientación sobre la aplicación de la Responsabilidad Social, este documento tiene varios alcances, entre los que se pueden citar algunos como el de asistir a las organizaciones en la orientación de sus políticas de responsabilidad social en lo que se refiere a las diferencias en materia cultural, ambiental, y legal, además de las condiciones económicas de desarrollo; poner énfasis en el rendimiento y mejora de los resultados, etc. 
Dentro de este marco ha de considerarse la responsabilidad social de las pequeñas y medianas empresas, en adelante PyMEs ${ }^{1}$ colombianas, se dice que la RSE se encuentra más ligada a las grandes empresas, pero su aplicación en las PyMEs se da de forma más natural, porque tiene una mayor relación con el entorno y su proximidad con los clientes, por lo que se podría decir que las PyMEs aplican acciones de RSE de forma habitual y que se hallan asociadas con su rutina diaria, sin muchas veces tener conocimiento sobre el concepto, pero lo más importante, es que sin la implicación de las PyMEs en la economía, la RSE no puede tener un impacto real en la sociedad (Solarte, 2013).

Por lo tanto, la RSE que aplican las PyMEs se encuentra en que desarrollan actividades sociales y comunitarias, pero lo realizan de manera informal (Roser, 2006). Según Cardona (2014), afirma que el 95.4\% de la industria nacional son PyMEs y que el $23 \%$ de ellas adquieren iniciativas de RSE, también menciona que hay una desinformación sobre la implementación de políticas de responsabilidad social empresarial en las pequeñas empresas, pero por ser la mayoría de empresas en Colombia PyMEs, estas tienen una gran incidencia sobre la reinserción en factor de empleabilidad.

Con respecto a la Responsabilidad Social Empresarial en el contexto de conflicto y posconflicto ${ }^{2}$, según Prandi y Lozano (2010) sostienen que es la relación conjunta de tres ámbitos: derechos humanos, desarrollo y paz, que se hallan interconectados entre sí y que esta conexión debe verse reflejada

${ }^{1}$ La Ley 560 de 2000 definió como PyMEs a toda unidad de explotación económica, realizada por persona natural o jurídica, en actividades empresariales, agropecuarias, industriales, comerciales o de servicios, rural o urbana, que cumpla los parámetros fijados por esta ley.

${ }^{2} \mathrm{Al}$ dar una definición clara de posconflicto se ha encontrado que según la Universidad de los Andes y la Fundación Ideas para La Paz, dedicados a las investigaciones sobre el conflicto armado en Colombia, definen también el posconflicto como "aquel periodo de tiempo que se inicia con el cese de hostilidades entre las partes previamente enfrentadas" (Rettberg A. 2002, p17), situación a la cual debería añadírsele el hecho de lograr superar las secuelas dejadas por el conflicto, de la mano del diseño de reformas sociales y políticas que permitan los cambios necesarios para que el proceso de posconflicto sea integral y exitoso.

Para dar un poco más de claridad frente al significado de posconflicto, los investigadores Ayala y Hurtado (2007), afirman que: "El posconflicto se construye, no se alcanza". De acuerdo a su teoría, "El posconflicto no puede entenderse como un fin último, sino como un proceso en el que hay varias tareas por hacer, entre ellas, el fortalecimiento -e incluso, la refundación del Estado y por ese camino, el logro de una legitimidad amplia y plural que reemplace el imaginario negativo que de este tienen amplios sectores de la nación". 
intrínsecamente en las políticas de Responsabilidad Social Empresarial, pero no solo en su contenido sino también en como dicha política se construye. Otra de las reflexiones de Prandi y Lozano (2010) es que la RSE no puede ir desligada del contexto en el que opera, sino que debe reflejar y dar respuesta a los retos humanos, tecnológicos, y medio ambientales, entre otros.

Así mismo, Prandi y Lozano (2010) plantean que las políticas de RSE en los ámbitos económicos y sociales que están dirigidos a la construcción de paz tienen dos objetivos: el primero, es económico, enfocado en crear oportunidades de subsistencia y emprendimiento y el segundo, es el social, para atender la exclusión social surgida a raíz del conflicto, de ahí, que mediante la RSE "las empresas pueden contribuir a reparar las fracturas sociales entre las comunidades enfrentadas propiciando actividades económicas conjuntas que deberán ser diseñadas con gran cautela y desde el conocimiento profundo del conflicto y de sus consecuencias (Prandi y Lozano 2010, p. 47).

De igual manera, los autores exponen que para alcanzar estos objetivos, las empresas deben actuar en las siguientes líneas (no excluyentes): Facilitar formación técnicay en valores (derechos humanos y paz); emplearo subcontratar a colectivos involucrados en el conflicto mediante un acompañamiento y una atención personalizada y forjar alianzas con las comunidades locales y sus organizaciones políticas, económicas y sociales, para así apoyar su desarrollo comunitario.

En suma, la Responsabilidad Social Empresarial implica la existencia de tres características fundamentales que son: primero, existe un criterio voluntario; segundo, incluyen dentro de sus actividades la preocupación social y medioambiental y tercera, debe tomar decisiones compartidas con todos los actores sociales de su entorno como son empleados, proveedores, el estado, clientes, consumidores, etc.

De otro lado, la Responsabilidad Social Empresarial se refiere a que a la visión general que se tiene del negocio, debe añadirse otros ingredientes tales como el respeto por los valores éticos, las personas, las comunidades y el medio ambiente, más específicamente luchar por el respeto de los derechos humanos; el cumplimiento de las normas laborales; la difusión de tecnologías inofensivas para el medio ambiente y la lucha contra la corrupción. 


\section{Inclusion laboral de poblacion reinsertada}

El ordenamiento jurídico colombiano determinó al trabajo como un derecho y como un deber. Es un derecho, porque debe ser amparado por el Estado a través de mecanismos legales y es un deber, porque constituye una obligación de las personas para con la sociedad en producir y aportar al proceso económico y social y al bienestar colectivo, por lo tanto, corresponde al Estado crear políticas sociales para la generación de empleo, estabilidad y desarrollo (Roldán, 2013). También el Estado cuenta con unos principios laborales constitucionales, en los cuales, se encuentra la igualdad de oportunidades ${ }^{3}$.

De ahí que es necesario comprender la importancia y alcance de la inclusión laboral y su relevancia dentro de la sociedad, especialmente con los más desfavorecidos, enmarcada dentro de un proceso más amplio que es la inserción social. En la medida en que el trabajo constituye un factor de integración social, debe entenderse la inserción laboral de aquellos más desfavorecidos (e incluso discriminados, como lo son los desmovilizados) desde dos aspectos: como un fin y como un medio en sí mismo. Un fin pues su objetivo final es darle al trabajador desfavorecido la posibilidad de acceder a estructuras normales y legales de empleo, y un medio a través del cual este sea considerado, reintegrado y auto considerado como parte de la sociedad (Cháves Ávila \& Sajardo Moreno, 1999 En Roldán, 2013, p. 113).

\footnotetext{
3 Se entiende por igualdad de oportunidades la misma disposición en abstracto frente a una eventual situación; es compartir la expectativa ante el derecho, así luego, por motivos justos, no se obtengan exactamente las mismas posiciones, o los mismos objetivos. Como todos los miembros de la especie humana comparten la identidad esencial, es lógica consecuencia que se compartan las mismas oportunidades." Corte Constitucional, sentencia C-023 de 1994 (27 de enero de 1994). Adicionalmente "La igualdad de oportunidades es, por consiguiente, un derecho fundamental mediante el que se "equipara" a las personas en inferioridad de condiciones, para garantizarles el pleno goce de sus derechos". Corte Constitucional, sentencia T-288 de 1995 (5 de julio de 1995).
} 


\section{Métodos}

La investigación que se realizó es de tipo cuantitativo descriptivo. Las variables a medir fueron: primero, el compromiso de los microempresarios con la generación de empleo y la implementación de políticas de responsabilidad social empresarial en el posconflicto y segundo, la divulgación de las políticas del gobierno que buscaban un empresario involucrado en los planes de solución del posconflicto.

La población objeto de estudio fueron 20 microempresas ubicadas en la ciudad de Bogotá. La investigación se enfocó en las empresas PyMEs, por varias razones: la primera, tienen un papel muy importante en la creación de empleo y en el desarrollo económico; segundo, por su proximidad a las comunidades en donde desarrollan su actividad, crean un alto impacto y tercero, de alguna manera, son indefensas ante las grandes empresas.

Para el desarrollo de la investigación se seleccionó como instrumento de recolección de datos una encuesta, en la que se plantearon seis preguntas cerradas, las cuales, miden las variables que se determinaron para identificar la participación y la voluntad de los microempresarios, al igual que el gobierno en términos de responsabilidad social empresarial en el marco del posconflicto. 


\section{Resultados}

De la encuesta realizada, la información se procesó mediante la tabulación en Excel, arrojando los siguientes resultados (Tabla 1):

Tabla 1. Tabulación de la encuesta

\begin{tabular}{|c|c|c|c|c|c|}
\hline $\begin{array}{c}\text { No. } \\
\text { Pregunta }\end{array}$ & PREGUNTAS & SI & NO & $\begin{array}{c}\text { NO } \\
\text { SABE/NO } \\
\text { RESPONDE } \\
\end{array}$ & $\begin{array}{c}\text { ACOMPAÑAR } \\
\text { Y } \\
\text { APOYAR } \\
\end{array}$ \\
\hline 1 & $\begin{array}{l}\text { ¿Conoce usted de políticas } \\
\text { gubernamentales que } \\
\text { promuevan, apoyen o } \\
\text { incentiven a las empresas en } \\
\text { la generación de empleo para } \\
\text { las personas reinsertadas? }\end{array}$ & & $85 \%$ & $15 \%$ & \\
\hline 2 & $\begin{array}{l}\text { ¿En su empresa, contrataría } \\
\text { usted personas reinsertadas de } \\
\text { las FARC? }\end{array}$ & $90 \%$ & $10 \%$ & $0 \%$ & \\
\hline 3 & $\begin{array}{l}\text { ¿Priorizaría la empresa la } \\
\text { oferta de empleos para las } \\
\text { mujeres reinsertadas? }\end{array}$ & $100 \%$ & $0 \%$ & $0 \%$ & \\
\hline 4 & $\begin{array}{l}\text { ¿Incentivaría su decisión de } \\
\text { contratar personal reinsertado } \\
\text { si el gobierno nacional } \\
\text { implementa estímulos } \\
\text { tributarios a las empresas que } \\
\text { generen empleo para esta } \\
\text { población? }\end{array}$ & $100 \%$ & $0 \%$ & $0 \%$ & \\
\hline 5 & $\begin{array}{l}\text { ¿Cuenta dentro de su } \\
\text { compañía con políticas } \\
\text { de responsabilidad social } \\
\text { empresarial? }\end{array}$ & $20 \%$ & $70 \%$ & $10 \%$ & \\
\hline 6 & $\begin{array}{l}\text { ¿Está de acuerdo con que el } \\
\text { gobierno nacional sea el único } \\
\text { responsable de los procesos } \\
\text { sociales (capacitación, } \\
\text { acompañamiento psicosocial, } \\
\text { etc.)? }\end{array}$ & $0 \%$ & $50 \%$ & $0 \%$ & $50 \%$ \\
\hline
\end{tabular}

Fuente. Elaboración propia de los autores. 
De los resultados obtenidos en la encuesta, se dedujo lo siguiente:

En la pregunta 1, se observa que el $85 \%$ de las empresas encuestadas respondieron no y el $15 \%$ no saben o no responden, lo que arroja que definitivamente el $100 \%$ no conocen las políticas gubernamentales que promueven, apoyan o incentivan a las empresas en la generación de empleo para las personas reinsertadas.

En la pregunta 2, el $90 \%$ de las empresas sí contratarían personas reinsertadas de las FARC, mientras que el $10 \%$ no lo harían, lo que quiere decir que las PyMEs encuestadas están comprometidas casi por completo con la oferta de empleo para los desmovilizados de las FARC y su aporte en el posconflicto.

En la pregunta 3, todas las empresas encuestadas le dan prioridad a la oferta de empleos para las mujeres reinsertadas, lo cual indica que las empresas consideran a la mujer como uno de los personajes más importantes en la sociedad y le dan su lugar a la hora de evaluar a este tipo de población.

En la pregunta 4, todas las empresas incentivarían su decisión de contratar personal reinsertado si el gobierno nacional implementa estímulos tributarios como por ejemplo: exención de impuestos, reducción de tarifas de impuestos, entre otros, a las empresas que generen empleo para esta población.

En la pregunta 5, el 20\% de los encuestados responden que sí cuentan dentro de su compañía con políticas de responsabilidad social empresarial, el $70 \%$ responden que no y el $10 \%$ no saben, no responden, lo cual indica que no las conocen. Con respecto a esta pregunta, adicionalmente se les interrogó si han planeado incluir políticas para la población reinsertada, donde se evidenció que las empresas que cuentan con políticas de RSE, que son mínimas, no han pensado en incluir políticas encaminadas en la generación de empleo para el posconflicto.

Y en la pregunta 6 , el 50\% de las empresas respondieron que no están de acuerdo con que el gobierno nacional sea el único responsable de los procesos sociales (capacitación, acompañamiento psicosocial, etc.), mientras que el otro $50 \%$ consideran acompañar y apoyar al gobierno en dichos procesos, o sea, que 
las empresas coinciden en que el gobierno nacional no es el único responsable y que cada uno de ellos está dispuesto a aportar para las actividades necesarias en pro de la reinserción a la vida social de los excombatientes de las FARC, lo cual significa que definitivamente las PyMEs encuestadas están dispuestas a trabajar en conjunto con el gobierno para diseñar políticas de RSE que busquen mejorar la calidad de vida y propiciar ambientes sociales adecuados para la población reinsertada en el marco del posconflicto.

\section{Discusión}

Estos datos indican que el gobierno no ha hecho un esfuerzo suficiente en involucrar a las PyMEs en el compromiso de generación de políticas de RSE y en la preparación para la oferta de empleo en el posconflicto, lo cual coincide con Gómez (2010) quien dice que en Colombia, la RSE aún se encuentra en proceso de fortalecimiento a nivel empresarial y que tanto las directivas como los trabajadores ven la responsabilidad social desde otros puntos de vista, como son el bienestar, la capacitación y el apoyo a la comunidad, (pero esto aplicado a las grandes empresas, las cuales tienen más propiedad del tema), mientras que las PyMEs están hasta ahora iniciando el proceso de integrar las buenas prácticas dentro de su gestión estratégica.

También se evidencia que la mayoría de las PyMEs no tienen políticas de RSE y lo que es más preocupante, una importante porción de estas no saben qué es RSE, de forma que difícilmente podrán generarlas. Esto no significa que definitivamente no hagan nada por la sociedad, por sus empleados o por el medio ambiente, solo que no lo han determinado mediante políticas empresariales. También se refleja, que en su mayoría, las PyMEs en Colombia, son creadas por emprendedores a quienes les ha hecho falta formación empresarial y/o educativa y llevando a cabo las actividades de manera empírica.

Lo anterior converge con lo que declara Gómez (2010), que las PyMEs están interesadas en hacer prácticas de responsabilidad social empresarial, pero que el problema radica en que estos empresarios, primero, no saben cómo identificarlas y segundo, aún no tienen claro cómo se pueden involucrar y cómo la pueden incluir dentro de sus procesos, ni tampoco saben cuáles serán los resultados esperados. De ahí que se observa durante los últimos años que la 
responsabilidad social empresarial ha venido fortaleciéndose, pasando de una definición filosófica a mediciones de impacto.

Como se puede observar, estos resultados tienen sus implicaciones, debido a que las PyMEs colombianas hasta ahora están aprendiendo a incorporar dentro de sus actividades las estrategias de RSE, como son capacitar al capital humano, cumplir con la legislación nacional, establecer reglas claras frente a la limpia competencia, mejorar las relaciones con la comunidad, establecer las condiciones en la cadena de suministro, plantear otros escenarios frente al cuidado del medio ambiente y el desarrollo social sostenible, entre otros, por lo que es recomendable que las PyMEs reconozcan en la RSE uno de los componentes fundamentales para aumentar su productividad, debido a que las transforma en agentes de cambio frente a sus comunidades y a sus grupos de interés.

También se aprecian algunas limitaciones tales como deficiencias de la muestra, la cual fue demasiado pequeña y problemas de diseño en donde el cuestionario de la encuesta es muy exiguo y generalizado, lo cual generó dificultad en la interpretación de los resultados, haciendo más dificil determinar de manera más amplia la incidencia de la responsabilidad social de las PyMEs en el marco del posconflicto, como soporte para la inclusión laboral de la población reinsertada.

\section{Conclusión}

Se establece que la mayoría de las PyMEs no tienen políticas de RSE y algunas de ellas no saben qué es RSE, pero muchas la ejecutan de forma indirecta, capacitando a los empleados, cumpliendo con todos los compromisos con los grupos de interés y sobretodo, aportando al medio ambiente y a los problemas sociales, como la inclusión laboral de la población reinsertada en el marco del posconflicto. 


\section{Referencias bibliográficas}

Backman, J. (2012). La responsabilidad social en la Mipyme colombiana, desde una postura alternativa. Recuperado de: http:// www.cladea.org/ index.php?...responsabilidad-social...pyme-colombiana.

Cajiga J. (2011). El concepto de responsabilidad social Empresarial RSE. Centro Mexicano para la Filantropía (CEMEFI). Recuperado de: http:// www.cemefi.org/esr/images/stories/pdf/esr/concepto_esr.pdf.

Cárdenas P. (2002). Las PyMEs: Vitales para la recuperación del crecimiento económico. Recuperado de $:$ http://www.usergioarboleda.edu.co/PyMEs/ noticia8.htm.

Cardona, O. (2014). Director Ejecutivo de Alianzas por el Desarrollo En Las Pyme le apuestan a prácticas de Responsabilidad Social Empresarial (RSE). Recuperado de: http://www.rsnoticias.com >

CCRE (2004). Articulo CCRE - Construyendo Confianza: ¿Que es Responsabilidad Social Empresarial? Recuperado de: http://ww.ccre. org.co.

(2004). Articulo CCRE. Responsabilidad Social Empresarial, El Nuevo Paradigma Para Las PyMEs En La Región (Hugo Vergara Director Ejecutivo Forum EMPRESA). Recuperado de: http://ww.ccre. org.co.

Comisión de las comunidades europeas. (2001). Libro Verde. Fomentar un marco europeo para la responsabilidad social de las empresas. Bruselas: Documento COM 366 Final, p. 38. Recuperado de: http:// www.degerencia.com/.../que-es-la-responsabilidad-social-empresarial,

El Espectador. Un país de PyMEs. Artículo de prensa. Recuperado de: http://www.elespectador.com/noticias/economia/un-pais-de-PyMEsarticulo-285125. 
Gómez, L. F. (2010). Travesías y abordajes de la responsabilidad social. En Cadena, H. (2014). La responsabilidad social empresarial como fuente de competitividad en las PyMEs. Trabajo de grado. Universidad Militar Nueva Granada. Bogotá. 11-12

González, P. (2011). Directora de Responsabilidad Social Empresarial (RSE) de la Cámara de Comercio de Bogotá. PyMEs: barreras y retos para implementar estrategias de RSE. Recuperado de: http:// www. gestrategica.org/templates/noticias_detalle.php?id=502.

Inercia digital. (2015). Recuperado de: http://blog.inerciadigital.com/ wp-content/ uploads/2015/05/Fotolia_21277151_XS.jpg

Más Business. La Responsabilidad Social Empresaria en las PyMEs. Recuperado de: http://www.mas-business.com/docs/La\%20RSocial\%20 en\%20PyMEs.pdf.

Prandi, M. y Lozano, J. (2010). La RSE en contextos de conflicto y posconflicto: de la gestión del riesgo a la creación de valor (eds.). Bellaterra: Escola de Cultura de la Pau.

Reyno, M. (2000). Responsabilidad social empresarial (RSE) como ventaja competitiva. Recuperado de: http://www.eumed.net/libros.../ Concepto\%20de\%20responsabilidad\%20social.

Roldán, L.(2013). La inclusión laboral de los desmovilizados del conflicto en Colombia: auténtico mecanismo emancipador de la violencia en Colombia. Revista Univ. Estud. $N^{\circ}$ 10: 107-123, enero-diciembre. Pontificia Universidad Javeriana.

Roser, I. (2006). Guía de la RSC para las PyMEs. Responsabilidad social corporativa. Madrid. Fundación el Monte y observatorio para la RSC. P. 9. Recuperado de: http://www.observatoriorsc.org.

Solarte, R. (2013). La responsabilidad social en las PyMEs. Una nueva cultura de competitividad sostenible. Recuperado de: http://www.eben-spain. org/docs/Papeles/XII/Roberto_Solarte_Rodriguez.pdf. 
Vanguardia.com. (2011). La responsabilidad social empresarial no es solo para grandes empresas. Recuperado de: http://www.vanguardia.com/ economia/local/91451-la-responsabilidad-social-empresarial-no-essolo-para-grandes-empresas. 